\title{
Erratum: Noise correlations of the ultracold Fermi gas in an optical lattice [Phys. Rev. A 77, 053602 (2008)]
}

\author{
T. Paananen, T. K. Koponen, P. Törmä, and J.-P. Martikainen
}

(Q) (Received 12 February 2018; published 26 March 2018)

DOI: 10.1103/PhysRevA.97.039907

The finite-temperature correlations in Eq. (29) of our work had some mistakes. Finite-temperature correlations should be

$$
\begin{aligned}
G_{\uparrow \downarrow}\left(\mathbf{r}, \mathbf{r}^{\prime}\right)= & A(t)^{4} \sum_{\mathbf{k}}\left\{\delta\left(\mathbf{r}-\frac{\hbar t \tilde{\mathbf{k}}}{m}\right) \delta\left(\mathbf{r}^{\prime}+\frac{\hbar t\left(\tilde{\mathbf{k}}{ }^{\prime}-\mathbf{q}\right)}{m}\right)\right. \\
& \left.\times\left|u_{\mathbf{k}, \mathbf{q}}\right|^{2}\left|v_{\mathbf{k}, \mathbf{q}}\right|^{2}\left[f\left(E_{\uparrow, \mathbf{k}, \mathbf{q}}\right)+f\left(E_{\downarrow, \mathbf{k}, \mathbf{q}}\right)-1\right]\left[f\left(E_{\uparrow, \mathbf{k}, \mathbf{q}}\right)+f\left(E_{\downarrow, \mathbf{k}, \mathbf{q}}\right)-1\right]\right\}, \\
G_{\downarrow \uparrow}\left(\mathbf{r}, \mathbf{r}^{\prime}\right)= & A(t)^{4} \sum_{\mathbf{k}}\left\{\delta\left(\mathbf{r}+\frac{\hbar t(\tilde{\mathbf{k}}-\mathbf{q})}{m}\right) \delta\left(\mathbf{r}^{\prime}-\frac{\hbar t \tilde{\mathbf{k}}^{\prime}}{m}\right)\right. \\
& \left.\times\left|u_{\mathbf{k}, \mathbf{q}}\right|^{2}\left|v_{\mathbf{k}, \mathbf{q}}\right|^{2}\left[f\left(E_{\uparrow, \mathbf{k}, \mathbf{q}}\right)+f\left(E_{\downarrow, \mathbf{k}, \mathbf{q}}\right)-1\right]\left[f\left(E_{\uparrow, \mathbf{k}, \mathbf{q}}\right)+f\left(E_{\downarrow, \mathbf{k}, \mathbf{q}}\right)-1\right]\right\}, \\
G_{\uparrow \uparrow}\left(\mathbf{r}, \mathbf{r}^{\prime}\right)= & A(t)^{4} \sum_{\mathbf{k}}\left\{\delta\left(\mathbf{r}-\frac{\hbar t \tilde{\mathbf{k}}}{m}\right) \delta\left(\mathbf{r}^{\prime}-\frac{\hbar t \tilde{\mathbf{k}}}{m}\right)\left\{\left|u_{\mathbf{k}, \mathbf{q}}\right|^{2} f\left(E_{\uparrow, \mathbf{k}, \mathbf{q}}\right)+\left|v_{\mathbf{k}, \mathbf{q}}\right|^{2}\left[1-f\left(E_{\downarrow, \mathbf{k}, \mathbf{q}}\right)\right]\right\}\right. \\
& \left.\times\left(1-\left\{\left|u_{\mathbf{k}, \mathbf{q}}\right|^{2} f\left(E_{\uparrow, \mathbf{k}, \mathbf{q}}\right)+\left|v_{\mathbf{k}, \mathbf{q}}\right|^{2}\left[1-f\left(E_{\downarrow, \mathbf{k}, \mathbf{q}}\right)\right] t\right\}\right)\right\}, \\
G_{\downarrow \downarrow}\left(\mathbf{r}, \mathbf{r}^{\prime}\right)= & A(t)^{4} \sum_{\mathbf{k}}\left\{\delta\left(\mathbf{r}-\frac{\hbar t \tilde{\mathbf{k}}}{m}\right) \delta\left(\mathbf{r}^{\prime}-\frac{\hbar t \tilde{\mathbf{k}}}{m}\right)\left\{\left|u_{\mathbf{k}, \mathbf{q}}\right|^{2} f\left(E_{\downarrow, \mathbf{k}, \mathbf{q}}\right)+\left|v_{\mathbf{k}, \mathbf{q}}\right|^{2}\left[1-f\left(E_{\uparrow, \mathbf{k}, \mathbf{q}}\right)\right]\right\}\right. \\
& \left.\times\left(1-\left\{\left|u_{\mathbf{k}, \mathbf{q}}\right|^{2} f\left(E_{\downarrow, \mathbf{k}, \mathbf{q}}\right)+\left|v_{\mathbf{k}, \mathbf{q}}\right|^{2}\left[1-f\left(E_{\uparrow, \mathbf{k}, \mathbf{q}}\right)\right]\right\}\right)\right\} .
\end{aligned}
$$

At low temperatures this does not affect the results in our work. At higher temperatures qualitative behavior remains the same, but some numerical values might be different in the figures illustrating the behavior.

We thank K. Ala-Mattinen for bringing this to our attention. 